\title{
On Groups of Odd Order Admitting an Elementary 2-Group of Automorphisms
}

\author{
Karise G. Oliveira (*) - Pavel Shumyatsky $(* *)$ - Carmela Sica $(* * *)$
}

ABstRACT - Let $G$ be a finite group of odd order with derived length $k$. We show that if $G$ is acted on by an elementary abelian group $A$ of order $2^{n}$ and $C_{G}(A)$ has exponent $e$, then $G$ has a normal series $G=G_{0} \geq T_{0} \geq G_{1} \geq T_{1} \geq \cdots$ $\geq G_{n} \geq T_{n}=1$ such that the quotients $G_{i} / T_{i}$ have $\{k, e, n\}$-bounded exponent and the quotients $T_{i} / G_{i+1}$ are nilpotent of $\{k, e, n\}$-bounded class.

Dedicated to Professor Said Sidki on the occasion of his roth birthday

\section{Introduction}

Let $G$ be a group and $A$ a group of automorphisms of $G$. The subgroup of all elements of $G$ fixed by $A$ is usually denoted by $C_{G}(A)$. It is well-known that very often the structure of $C_{G}(A)$ has strong influence over the structure of the whole group $G$. The influence seems especially strong in the case where $G$ is a finite group of odd order and $A$ is an elementary abelian 2-group. It was shown in [5] that if $G$ is a finite group of derived length $k$ on which an elementary abelian group $A$ of order $2^{n}$ acts fixedpoint-freely, then $G$ has a normal series $G=N_{0} \geq \cdots \geq N_{n-1} \geq N_{n}=1$ all of whose quotients are nilpotent and the class of $N_{i-1} / N_{i}$ is bounded with a

(*) Indirizzo dell'A.: Instituto Federal de Educação, Ciência e Tecnologia de Goiás, Av. Universitária, s/n, Vale das Goiabeiras, CEP: 75400-000 Inhumas GO, Brazil.

E-mail: karisemat@gmail.com

(**) Indirizzo dell'A.: Department of Mathematics, Universidade de Brasilia, 70.919 Brasilia - DF, Brazil.

E-mail: pavel@mat.unb.br

(***) Indirizzo dell'A.: Dipartimento di Matematica, Università di Salerno, Via Ponte don Melillo, 84084 Fisciano (SA), Italy.

E-mail: csica@unisa.it 
function of $k$ and $i$ (see also [7] for a short proof of this result). In the present paper we further exploit the techniques developed in [7]. One of the obtained results is the following theorem.

THEOREM 1.1. Let $G$ be a finite group of odd order and of derived length $k$. Suppose that $G$ admits an elementary abelian group $A$ of automorphisms of order $2^{n}$ such that $C_{G}(A)$ has exponent $e$. Then $G$ has a normal series

$$
G=G_{0} \geq T_{0} \geq G_{1} \geq T_{1} \geq \cdots \geq G_{n} \geq T_{n}=1
$$

such that the quotients $G_{i} / T_{i}$ have $\{k, e, n\}$-bounded exponent and the quotients $T_{i} / G_{i+1}$ are nilpotent of $\{k, e, n\}$-bounded class.

The other result deals with the situation where $\gamma_{c}\left(C_{G}(a)\right)$ has exponent $e$ for all automorphisms $a \in A^{\#}$. Here $A^{\#}$ denotes the set of non-trivial elements of $A$ and $\gamma_{i}(H)$ stands for the $i$-th term of the lower central series.

THEOREM 1.2. Let $G$ be a finite group of odd order and of derived length $k$. Suppose that a four-group $A$ acts on $G$ in such way that $\gamma_{c}\left(C_{G}(a)\right)$ has exponent e for all $a \in A^{\#}$. Then $G$ has a normal series

$$
G=T_{4} \geq T_{3} \geq T_{2} \geq T_{1} \geq T_{0}=1
$$

such that the quotients $T_{4} / T_{3}$ and $T_{2} / T_{1}$ are nilpotent of $\{e, c, k\}$-bounded class and the quotients $T_{3} / T_{2}$ and $T_{1}$ have $\{e, c, k\}$-bounded exponent.

Throughout the paper we say that a group $G$ is nilpotent of class $c$ meaning that the class of $G$ is at most $c$ and we say that $G$ has exponent $e$ meaning that the exponent of $G$ divides $e$. The expression " $(a, b, \ldots)$ bounded" stands for "bounded from above by a function depending only on the parameters $a, b, \ldots$.

\section{Auxiliary Results}

The following lemmas are well-known (see for example Theorem 6.2.2, Theorem 6.2.4 and Theorem 10.4.1 in [1]).

LEMMA 2.1. Let $G$ be a finite group admitting a coprime group of automorphisms A. Then we have 
a) $C_{G / N}(A)=C_{G}(A) N / N$ for any $A$-invariant normal subgroup $N$ of $G$;

b) $G=C_{G}(A)[G, A]$;

c) $[G, A]=[G, A, A]$;

d) If $G$ is abelian, then $G=C_{G}(A) \times[G, A]$.

LEMma 2.2. Let $G$ be a finite group admitting an abelian non-cyclic coprime group of automorphisms $A$. Then $G=\left\langle C_{G}(a) \mid a \in A^{\#}\right\rangle$.

LEMma 2.3. Let $G$ be a finite group of odd order admitting an automorphism a of order 2 such that $G=[G, a]$. Suppose that $N$ is an a-invariant normal subgroup of $G$ such that $C_{N}(a)=1$. Then $N \leq Z(G)$.

Our next lemma is immediate from [3, Lemma 2.6].

LEMMA 2.4. Let $G$ be a finite group admitting a coprime group of automorphisms A. Suppose that $G$ is generated by a family $\left\{H_{i} \mid i \in I\right\}$ of normal A-invariant subgroups. Then

$$
C_{G}(A)=\left\langle C_{H_{i}}(A) \mid i \in I\right\rangle .
$$

LEMMA 2.5. Let $G$ be a metabelian finite group of odd order admitting an automorphism a of order 2 such that $C_{G}(a)$ has exponent $e$ and $G=[G, a]$. Then $G^{\prime}$ has exponent e.

Proof. Since $G / G^{\prime}$ is abelian and $G=[G, a]$, by Lemma 2.1 $C_{G / G^{\prime}}(a)=1$ and so $C_{G}(a) \leq G^{\prime}$. Let $N$ be the normal closure of $C_{G}(a)$ in $G$. Since $G^{\prime}$ is abelian we conclude that $N$ has exponent $e$. On the other hand, $G / N$ is abelian because $a$ acts on $G / N$ fixed-point-freely. Therefore $G^{\prime}=N$ and the result follows.

Proposition 2.6. Let $G$ be a finite group of odd order and of derived length $k$ admitting an automorphism a of order 2 such that $C_{G}(a)$ has exponent e and $G=[G, a]$. Then $G^{\prime}$ has $\{e, k\}$-bounded exponent.

Proof. In view of Lemma 2.5 the result is obvious if $k \leq 2$. Suppose that $k \geq 3$ and let $M=G^{(k-1)}$. By induction we conclude that $G^{\prime} / M$ has $\{e, k\}$-bounded exponent. Hence, it is enough to show that $M$ has $\{e, k\}-$ bounded exponent. Working with the quotient $G /\left\langle C_{M}(a)^{G}\right\rangle$ we can simply assume that $C_{M}(a)=1$. Lemma 2.3 now shows that $M \leq Z(G)$. Therefore $G^{(k-2)}$ is nilpotent of class 2 . Since $G^{(k-2)} / M$ has $\{e, k\}$-bounded exponent, it follows that $G^{(k-2)}$ has $\{e, k\}$-bounded exponent [4, Theorem 2.5.2]. In particular, $M$ has $\{e, k\}$-bounded exponent. 
A well-known theorem of Hall says that if $G$ is a soluble group of derived length $k$ and all metabelian sections of $G$ are nilpotent of class at most $c$, then $G$ is nilpotent of $\{k, c\}$-bounded class [2]. We will require the following related result obtained in [6]. We denote by $f(g, c)$ the expression $(g-1) \frac{c(c+1)}{2}+c$.

THEOREM 2.7. Let $G$ be a group and $N$ a nilpotent normal subgroup of $G$ of class $g$ such that $\gamma_{c+1}\left(G / N^{\prime}\right)$ has exponent e. Suppose that $\gamma_{c+1}(G)$ is soluble of derived length $d$. Then $\gamma_{f(g, c)+1}(G)$ has finite $\{g, e, c, d\}$-bounded exponent.

The next proposition was obtained in [7, Corollary 3.2]. It plays a crucial role in the subsequent proofs.

Proposition 2.8. There exists a number $s=s(k, n)$ depending only on $k$ and $n$ with the following property. Suppose that $G$ is a finite group of odd order that is soluble with derived length at most $k$. Assume that an elementary group $A$ of order $2^{n}$ acts on $G$ and let $R$ be a normal $A$ invariant subgroup of $G$ such that $C_{R}(A)=1$. Set $N=\bigcap_{a \in A^{\#}}[G, a]$. Then $[R, \underbrace{N, \ldots, N}_{s}]=1$.

\section{Main Results}

Proposition 3.1. Let $G$ be a finite group of odd order and of derived length $k$. Suppose that $G$ admits an elementary abelian group of automorphisms $A$ of order $2^{n}$ such that $C_{G}(A)$ has exponent $e$. Then $\bigcap_{a \in A^{*}}[G, a]$ is an extension of a group of $\{k, e, n\}$-bounded exponent by a nilpotent group of $\{k, e, n\}$-bounded class.

Proof. Set $N=\bigcap_{a \in A^{\#}}[G, a]$ and use induction on the derived length of $N$. If $N$ is abelian, the result is trivial. Suppose that the derived length of $N$ is greater than or equal to 2 and let $L$ be the metabelian term of the derived series of $N$. Put $M=L^{\prime} \cap C_{G}(A)$ and let $D$ be the normal closure of $M$ in $G$. Since $D$ is abelian with generators of order $e$, we see that $D$ has exponent $e$. Considering the quotient $G / D$ we can simply suppose that $C_{L^{\prime}}(A)=1$. Proposition 2.8 shows that $L^{\prime} \subseteq Z_{s}(L)$ and so $L$ is nilpotent of class $s+1$. By 
induction, $N / L^{\prime}$ is an extension of a group of $\{k, e, n\}$-bounded exponent by a nilpotent group of $\{k, e, n\}$-bounded class, say $c$. Since the derived length of $\gamma_{c+1}(N)$ is at most $k$, by Theorem 2.7 we conclude that $\gamma_{f(s+1, c)+1}(N)$ has $\{k, e, n\}$-bounded exponent, as required.

Given a group $H$ and positive integers $e$ and $c$, we denote by $V(H)$ the verbal subgroup of $H$ corresponding to the group-word $\left[x_{1}, \ldots, x_{c}\right]^{e}$ and by $W(H)$ the verbal subgroup of $H$ corresponding to the group-word $\left[x_{1}, \ldots, x_{3 c-2}\right]^{e^{3}}$.

LEMMA 3.2. Let $G$ be a finite metabelian group of odd order admitting a four-group of automorphisms A such that $\gamma_{c}\left(C_{G}(a)\right)$ has exponent e for all $a \in A^{\#}$. Then there exists a normal subgroup $M$ of $G$ such that $C_{M}(A)=1$ and $\gamma_{3 c-2}(G / M)$ has exponent $e^{3}$.

Proof. For every $a \in A^{\#}$ put $G_{a}=G^{\prime} C_{G}(a)$. It is easy to see that $\left[G_{a}, a\right]=\left[G^{\prime}, a\right]$ is normal in $G_{a}$. Since $G_{a} /\left[G^{\prime}, a\right]$ is isomorphic to a quotient of $C_{G}(a)$, it follows that $V\left(G_{a}\right)$ is contained in $\left[G^{\prime}, a\right]$. Since $G^{\prime}$ is abelian, by Lemma 2.1 we have $\left[G^{\prime}, a\right] \cap C_{G}(a)=1$. It follows that $V\left(G_{a}\right) \cap C_{G}(a)=1$. Let $M=\prod_{a \in A^{\#}} V\left(G_{a}\right)$, obviously $M$ is a normal subgroup of $G$. By Lemma 2.4, $C_{M}(A)=\left\langle C_{V\left(G_{a}\right)}(A) \mid a \in A^{\#}\right\rangle$ and so $C_{M}(A)=1$. By Lemma 2.2 we have $G=\left\langle G_{a} \mid a \in A^{\#}\right\rangle$. Thus, $G / M$ is product of three normal subgroups each of which is an extension of a group of exponent $e$ by a nilpotent group of class $c-1$. Thus $G / M$ is an extension of a group of exponent $e^{3}$ by a nilpotent group of class $3 c-3$.

LEMma 3.3. Let $G$ be a metabelian group such that $\gamma_{c}(G / Z(G))$ has exponent e. Then $\gamma_{c+1}(G)$ has exponent e.

Proof. Let $E=\gamma_{c}(G)$. Then $E /(Z(G) \cap E)$ has exponent $e$. For arbitrary elements $x_{1}, x_{2}, \ldots, x_{c}, x_{c+1} \in G$ we have

$$
\left[x_{1}, x_{2}, \ldots, x_{c}\right] \in E \text {. }
$$

Therefore

$$
\left[x_{1}, x_{2}, \ldots, x_{c}\right]^{e} \in Z(G) \cap E,
$$

whence

$$
\left[\left[x_{1}, x_{2}, \ldots, x_{c}\right]^{e}, x_{c+1}\right]=1 .
$$

Since $\left[x_{1}, x_{2}, \ldots, x_{c}\right] \in G^{\prime}$, which is a normal abelian subgroup of $G$, we 
obtain that

$$
\left[x_{1}, x_{2}, \ldots, x_{c}, x_{c+1}\right]^{e}=\left[\left[x_{1}, x_{2}, \ldots, x_{c}\right]^{e}, x_{c+1}\right]=1 .
$$

Hence, $\gamma_{c+1}(G)$ has exponent $e$.

Proposition 3.4. Let $G$ be a finite group of odd order and of derived length $k$ admitting a four-group of automorphisms $A$ such that $\gamma_{c}\left(C_{G}(a)\right)$ has exponent e for all $a \in A^{\#}$. Then $\bigcap_{a \in A^{\#}}[G, a]$ is an extension of a group of $\{e, c, k\}$-bounded exponent by a nilpotent group of $\{e, c, k\}$-bounded class.

Proof. Let $N=\bigcap_{a \in A^{\#}}[G, a]$. We use induction on the derived length of $N$. If $N$ is abelian the result is trivial. Suppose that the derived length of $N$ is greater than or equal to 2 and let $L$ be the metabelian term of the derived series of $N$. First we will show that $L$ is an extension of a group of $\{e, c, k\}-$ bounded exponent by a nilpotent group of $\{e, c, k\}$-bounded class. By Lemma 3.2 there exists a normal subgroup $M$ of $L$ such that $C_{M}(A)=1$ and $\gamma_{3 c-2}(L / M)$ has exponent $e^{3}$. Thus $W(L) \subseteq M$ and so $W(L) \cap C_{L}(A)=1$. If $W(L)=1$, then $\gamma_{3 c-2}(L)$ has exponent $e^{3}$. Suppose that $W(L) \neq 1$. Since $W(L) \cap C_{G}(A)=1$ it follows from Proposition 2.8 that there exists a bounded number $s$ such that

$$
[W(L), \underbrace{N, \ldots, N}_{s}]=1 .
$$

Let $t$ be the smallest number such that $W(L) \subseteq Z_{t}(N)$. We know that $t \leq s$. It will be shown by induction on $t$ that $L$ is an extension of a group of $\{e, c, k\}$-bounded exponent by a nilpotent group of $\{e, c, k\}$-bounded class. For $t=1$ we have the inclusion $W(L) \subseteq Z(N)$. In this case $L /(L \cap Z(N))$ is an extension of a group of exponent $e^{3}$ by a nilpotent group of class $3 c-3$. By Lemma 3.3 we deduce that $\gamma_{3 c-1}(L)$ has exponent $e^{3}$, as required.

Suppose now that $t \geq 2$ and the result is valid for $t-1$. Let $K=[W(L), \underbrace{N, \ldots, N}_{t-1}]$. It is clear that $K \leq Z(N)$. By induction it follows that $L / K$ is an extension of a group of $\{e, c, k\}$-bounded exponent by a nilpotent group of $\{e, c, k\}$-bounded class and by Lemma 3.3 also $L$ is an extension of a group of $\{e, c, k\}$-bounded exponent by a nilpotent group of $\{e, c, k\}$-bounded class, say $c_{1}$. Put $H=\gamma_{c_{1}+1}(L)$. So $H$ has $\{e, c, k\}$-bounded exponent. Passing to the quotient $G / H$ we can simply 
assume that $H=1$. By induction on the derived length of $N$, we deduce that $N / L^{\prime}$ is an extension of a group of $\{e, c, k\}$-bounded exponent by a nilpotent group of $\{e, c, k\}$-bounded class, say $c_{2}$. By Theorem 2.7 we conclude that $\gamma_{f\left(c_{1}, c_{2}\right)+1}(N)$ has $\{e, c, k\}$-bounded exponent, as required.

Now we are in a position to prove our main results.

Proof of Theorem 1.1. If $n=1$, Proposition 2.6 guarantees that $[G, a]^{\prime}$ has $\{e, k\}$-bounded exponent and so

$$
G=G_{0} \geq[G, a]=T_{0} \geq[G, a]^{\prime}=G_{1} \geq T_{1}=1
$$

is the required series. Suppose that $n \geq 2$ and use induction on $n$. For every normal $A$-invariant subgroup $N$ of $G$ the group $A$ induces a group of automorphisms of $G / N$. In particular $A$ induces a group of automorphisms $B_{a}$ of $G /[G, a]$ for every $a \in A^{\#}$ with $\left|B_{a}\right| \leq 2^{n-1}$ and $C_{G /[G, a]}\left(B_{a}\right)$ of exponent $e$. Let $B$ be the elementary abelian group of order $2^{n-1}$. We can define an action of $B$ on $G /[G, a]$ as follows. For all $a \in A^{\#}$ we consider $B_{a}$ as a subgroup of $B$ and we can write $B=B_{a} \times D_{a}$ for a suitable subgroup $D_{a}$ of $B$. For any $b \in B$ there exists a unique pair $\left(b_{1}, b_{2}\right) \in B_{a} \times D_{a}$ such that $b=b_{1} b_{2}$. Then for any $x \in G /[G, a]$ we put $x^{b}=x^{b_{1}}$. This action is well defined and $C_{G /[G, a]}(B)$ has exponent $e$. Let $K=\prod_{a \in A^{\#}} G /[G, a]$, we have an action of $B$ on $K$ that extends the action of $B$ on every factor $G /[G, a]$ and $C_{K}(B)$ has exponent $e$.

By induction, $K$ has a series of length $2(n-1)$ with the required properties. By Proposition 3.1 the subgroup $N=\bigcap_{a \in A^{\#}}[G, a]$ is an extension of a group of $\{k, e, n\}$-bounded exponent by a nilpotent group of $\{k, e, n\}$ bounded class. Since $G / N$ embeds in $K$ the result follows.

Proof of Theorem 1.2. Each factor $G /[G, a]$ is isomorphic to a quotient of $C_{G}(a)$, so it is an extension of a group of exponent $e$ by a nilpotent group of class $c-1$. Therefore $K=\prod_{a \in A^{\#}} G /[G, a]$ is an extension of a group of exponent $e^{3}$ by a nilpotent group of class $3 c-3$. Let $N=\bigcap_{a \in A^{\#}}[G, a]$. Since $G / N$ embeds in $K$, we conclude that $\gamma_{3 c-2}(G / N)$ has exponent $e^{3}$. Proposition 3.4 ensures that $N$ is an extension of a group of $\{e, c, k\}-$ bounded exponent by a nilpotent group of $\{e, c, k\}$-bounded class. The proof is complete. 


\section{REFERENCES}

[1] D. Gorenstein, Finite Groups, Harper and Row, New York, Evanston, London, 1968.

[2] P. HaLl, Some sufficient conditions for a group to be nilpotent, Illinois J. Math., 2 (1958), pp. 787-801.

[3] B. Hartley, Periodic locally soluble groups containing an element of prime order with Černikov centralizer, Quart. J. Math. Oxford Ser., (2), 33, n. 131 (1982), pp. 309-323.

[4] E. KHUKHRo, Nilpotent groups and their automorphisms, Berlin, New York, De Gruyter expositions in mathematics; 8 (1993).

[5] P. Shumyatsky, Groups with regular elementary 2-groups of automorphisms, Algebra and Logic, 27, no. 6 (1988), pp. 447-457.

[6] P. Shumyatsky, On extensions of groups of finite exponent, Glasgow Math J., 45, n. 3 (2003), pp. 535-538.

[7] P. ShumYatsky - C. SicA, On groups admitting a fixed-point-free elementary 2-group of automorphisms, Comm. Algebra, 38, n. 11 (2010), pp. 4188-4192.

Manoscritto pervenuto in redazione il 24 febbraio 2011. 